\title{
CONFUSIONES DE SIBILANTES EN EL MANUSCRITO 20241/13 DE LA BIBLIOTECA NACIONAL DE MADRID
}

\author{
Pilar Montero Curiel \\ Universidad de Extremadura
}

El manuscrito 20241/13 de la Biblioteca Nacional de Madrid es un cuadernillo de 28 folios que recoge las respuestas dadas por Don Francisco Sánchez y Santiago, párroco de la villa de Alburquerque (Badajoz), a Don Tomás López, Geógrafo del rey Carlos IV, para completar un interrogatorio de quince preguntas orientado a la composición de un Diccionario Geográfico de España. La redacción del texto se ajusta a las quince preguntas que componen el cuestionario remitido por Tomás López a los obispos, párro$\cos$ y autoridades de la época, y proporciona noticias sobre la situación administrativa del lugar al que se refiere, las distancias y comunicaciones, los accidentes geográficos, la toponimia menor, la flora y la fauna, la época de fundación, los derechos fiscales, los recursos municipales, las actividades agrícolas y ganaderas, la sanidad, la instrucción pública y otros asuntos de interés que ayudan a completar el estudio geográfico para el que se elaboran las consultas. Las respuestas dadas a estos cuestionarios han servido a los geógrafos e historiadores como fuente de información para conocer la visión que de su propia época tenían los españoles del siglo XVIII.

Los cuestionarios de Tomás López, hasta la fecha, se han analizado en el contexto de los interrogatorios del siglo xVIII que pretenden recabar infornación de carácter enciclopédico - en el sentido más amplio del términoara preparar un Diccionario Geográfico de España que el propio autor no . .do ver impreso en vida ${ }^{1}$. Hoy en día, la Biblioteca Nacional de Madrid

\footnotetext{
I Por lo menos en su totalidad, ya que en el año 2000 Ediciones Trigo ha editado en Madrid un facsímil del volumen que apareció en 1763 con el título Descripcion de la provincia de Madrid. Por Thomas Lopez, Pensionista de S. M. y de la Real Academia de San Fernando, Madrid. Por Joachin Ibarra, calle de las Urosas. Año de 1763.
}

RFE, LXXXII, 2002, 1. $\div 2 .^{\circ}$, págs, 45-61 
guarda en sus fondos los manuscritos que los párrocos enviaron al cartógrafo real y, entre ellos, una serie muy extensa de hojas que contienen las descripciones de "la Provincia de Extremadura". A esta serie corresponde el manuscrito 20241/13 perteneciente al municipio de Alburquerque, localidad situada a 45 kilómetros al norte de Badajoz en la frontera con Portugal, cuyos folios van a servir para llevar a cabo un análisis de carácter filológico. Dicho manuscrito cuenta con una edición moderna que vio la luz en 1991, dentro del volumen titulado Estremadura. Por Lopez. Año de 1798 , a cargo de Gonzalo Barrientos Alfageme ${ }^{2}$. Por las fechas del manuscrito y por el lugar en el que se escribe, resulta interesante examinar el sistema fonológico que refleja, un sistema que muestra todos los elementos del castellano de la época y en el que, seguramente por la cercanía de Alburquerque con Portugal ${ }^{3}$, se descubren casos de seseo, ultracorrecciones que tienden a evitar el seseo y correcciones hechas sobre el mismo manuscrito, pruebas de una conciencia lingüística y un afán de perfección singulares.

\section{LAS GRAFIAS}

El cuaderno firmado por el párroco don Francisco Sánchez y Santiago se redacta a finales del siglo XVIII en Alburquerque (Badajoz), y encierra interesantes testimonios de seseo en un lugar en el que la continuidad del fenómeno está asegurada hoy en todos los registros, como ponen de relieve los mapas del Atlas Lingüistico de la Peninsula Ibérica, publicados en 1962, y las encuestas realizadas en el mes de febrero de 2000 a un grupo

\footnotetext{
${ }^{2}$ La transcripción de los textos (que en esta ocasión ha sido muy útil para cotejar las páginas dedicadas a Alburquerque con sus manuscritos originales) fue realizada por Maria del Carmen Sánchez y María Luz Gómez. Vid. Gonzalo Bartientos Alfageme (ed.), Estremadura. Por Lopez. Año de 1798, Mérida, Asamblea de Extremadura, 1991, pág. 12. Quiero agradecer al profesor Gonzalo Barrientos la ayuda prestada en los primesos pasos de este trabajo, al haber puesto a mi disposición sus ficheros sobre la localidad de Alburquerque y otros pueblos extremeños fronterizos con Portugal. Y a Leo v. Caprivi, que me prestó su tiempo para descifrar conmigo, palabra por palabra, cada línea del manuscrito.

${ }^{3}$ Como indica Juan M. Carrasco González, en la Edad Media "el señorío de Alburquerque (...) [es un] elemento de gran importancia en la más antigua influencia del portugués en ja Baja Extremadura y del castellano en las regiones vecinas de Portugal". Cfr. sus trabajos titulados «Hablas y dialectos portugueses o galaico-portugueses en Extremadura. (Parte I: Grupos dialectales. Clasificación de las hablas de Jálama)», en Anuario de Estudios Filológicos, XIX, 1996, págs. 135-148 y «Hablas y dialectos portugueses o galaicoportugueses en Extremadura. (Parte II y ültima: Otras hablas fronterizas. Conclusiones)m, en Anuario de Estudios Filológicos, XX, 1997, págs. 61-79. La referencia se encuentra en el articulo II, págs. 68-69.
} 
de hablantes del municipio extremeño ${ }^{4}$, que sesean con regularidad en su conversación cotidiana.

En el texto que nos ocupa la distinción entre consonantes sordas y sonoras no tiene ya ninguna relevancia fonológica; pero el seseo, que se da al lado de la distinción entre el fonema palatal fricativo sordo $/ \mathrm{s} / \mathrm{y}$ el fonema interdental fricativo sordo $/ \theta /$ y las reiteradas ultracorrecciones que originan casos de ceceo en el texto (al menos en el nivel gráfico), son detalles que llevan a pensar en la importancia que adquiere en el español hablado en Alburquerque un fenómeno como el seseo que bien podría tomarse como indicio de la influencia que la fonética de la lengua portuguesa ejerce sobre la castellana en los límites políticos entre las dos lenguas.

Como es bien sabido, en los albores del siglo XIX el sistema gráfico del español quedó prácticamente fijado con los signos que hoy empleamos, gracias a las reformas promovidas, en diferentes etapas desde 1726 hasta 1815 , por la Real Academia 5 . Así, en el español peninsular el signo $x$, a partir de la octava edición de la Ortografía académica (1815), dejó de servir para la representación del fonema velar fricativo sordo que hoy transcribimos como $j$ y quedó reservado para dar forma escrita a la pronunciación del grupo culto $k s$ (examen, exiguo) o a la $x$ latina del prefijo ex- (exordio, extremo $)^{6}$. El manuscrito de Alburquerque refleja con claridad, como era de esperar por su fecha de redacción, este sistema, aunque con alternancias que hablan de la falta de fijación gráfica u ortográfica de algunos signos: $B a$ daxoz, topónimo del obispado y del partido al que pertenece Alburquerque, se cita quince veces en el texto, siempre con la grafia $x$; esta grafia sirve para representar el mismo fonema velar fricativo sordo en exercicio (folios $1 \mathrm{r}, 2 \mathrm{v}$ ), exercer y otras variantes conjugadas de este verbo (folios $2 \mathrm{tr}, 21 \mathrm{v}$, $22 \mathrm{r}$ ), executivas $(22 \mathrm{r})$, exercitos $(17 \mathrm{r}, 18 \mathrm{r}, 8 \mathrm{v})$, fixados $(16 \mathrm{r})$ y fixo (20r), que tienen la grafia $x$ en latín para representar el grupo ks y que en castellano han evolucionado hacia el fonema $/ \mathrm{x} /$, velar fricativo sordo. También se encuentra la grafia $x$ en la expresión "Callexa de Limones" (10r), en los fitónimos carquexa (11r) y coscoxa (23r), términos que en sus étimos latinos

\footnotetext{
${ }^{4}$ Los individuos encuestados en Alburquerque en febrero del año 2000 son M. Berrocal García, de 75 años; J. García Rivero, de 55 años; S. Gibello Vélez, de 73 años y J. Rodriguez Caldera, de 68, todos ellos naturales y vecinos de Alburquerque. El cuestionario utilizado fue el del Ailas Lingüistico de España y Portugal (Madrid, Seminario de Geografia Lingüística del CSIC, 1974), en especial las preguntas sobre fonética, y una lista con las diecinueve voces del $A L P I$ que proporcionaban en los mapas de 1962 testimonios de seseo.

${ }^{5}$ Vid. Rafael Lapesa, Historia de la lengtta española, Madrid, Gredos, 1985, § 103, pág. 424. Para situar estas reformas ortográficas en su marco histórico, vid. el capitulo IIl del libro de Francisco Marcos Marín, Reforma y modernización del español, Madrid, Cátedra, 1979, págs. $77-133$.

${ }^{6}$ Vid. Rafael Lapesa, ibídem, $\S 102$, pág. 422.
} 
contenían el grupo LY, así como en el topónimo Trujillo, cuyo origen remite a la raíz prerromana *turga desde la variante latina TURGALIUM ${ }^{7}$. A la inclinación cultista del párroco pueden responder las formas expecificara $(9 \mathrm{v})$, expecie (19 v) y expecificada (23v), tal vez por analogia con otras palabras que comienzan por ex. La grafia $j$ domina en términos como ajos (2r), cajas (3r), "enla Torre del Omenaje" (3r), jurisdicción (1r), Jesuchristo $(3 \mathrm{r})$, obejas (2r), Juan (2v), vajo (3r), viejos (2v), entre otros muchos.

Además, la grafia $s s$ se mantiene con claridad en las formas adverbiales assi y assimismo, repartidas por todo el texto, y en superlativos formados con el sufijo latino -issimo, en alternancia con las formas modernas que han simplificado la geminada de acuerdo con los preceptos ortográficos de 1763, "que suprimen la distinción entre $-s s-$ y $-s$ - generalizando la $-s-"$ : "hombres eminentissimos" (15v) al lado de los "Excelentisimos Señores" (16r), el "Excelentisimo Señor Don Pedro Rodriguez Campomanes" (18v), el "Excelentisimo Señor Duque de Alburquerque" (21r) o el "Serenisimo Señor Infante de España" (6v). En el folio $21 \mathrm{r}$ se mezclan, en la misma estructura, las formas religiosso y religioso, sin diferencias entre la $s$ sorda y la sonora, dato irrelevante, por otra parte, en un texto castellano de finales del siglo XviI, si se tiene en cuenta que esta lengua redujo las diferencias entre la $s$ sorda y la sonora hacia mediados del siglo XIV y que las zonas que la han conservado en el norte de Extremadura y el Sur de Salamanca ${ }^{9}$ quedan muy lejos de Alburquerque, por mucho que Portugal, en la misma frontera, haya mantenido las diferencias hasta nuestros días.

Al lado de estos ejemplos, el texto muestra algunas anomalias producidas por tendencias cultas que utilizaban, hasta fechas recientes, transcripciones como "Sn. Matheo" (folio 1r, 1v), "quatro thenientes" (lv), "Jesuchristo" (3r), "Thesorero de la Cathedral" (16v), "Fray Bartholomé de Alburquerque" (16v), "Theniente Coronel de Cavalleria" (18r), "lector de fílosophia" (21r) y otras formas similares con las que, como explica Lapesa ${ }^{10}$, la Real Academia Española mantuvo una actitud conservadora con preferencia por el criterio etimológico frente al valor real de los signos ortográficos.

\footnotetext{
7 Vid. José Antonio Redondo Rodriguez y Pedro Juan Galán Sánchez, «El topónimo cacereño Trujillo: origen y evolución fonética», en Alcántara. Revista del Seminario de Estudios Cacereños, 12, 1987, págs. 105-113.

${ }^{8}$ Vid. Rafael Lapesa, op. cit., \$ 102, pág. 423.

${ }^{9}$ Como estudió Aurelio M. Espinosa (hijo), Arcaísmos dialectales, La conservación de "s)" y "zz" sonoras en Cáceres y Salamanca, Anejo XIX de la RFE, 1935. Cfr., para la pervivencia del fenómeno, el artículo de Manuel Ariza y Antonio Salvador, "Sobre la conservación de sonoras en la provincia de Cáceres», en Zeitschrift für romanische Philologte, 108, 3-4, 1992, págs. 276-292.

${ }_{10}$ Rafael Lapesa, op. cit., § 102, pág. 423.
} 
De igual forma, el manuscrito refleja las coincidencias fonéticas entre $b$ y $v$ (obejas, $2 \mathrm{r}$; vendize $3 \mathrm{v}$ ), entre $j$ y $g$ delante de las vocales palatales (Correjidor, 3r; privilegios, 2r; coje 7v; orijen, 9r), y entre y e $i$ (cuio, 5r, cuios $24 \mathrm{r}$, trayendose $6 \mathrm{r}$, reyertas $7 \mathrm{v}$, concluye $10 \mathrm{r}$, leyes $17 \mathrm{v}$, ganado yeguar $17 \mathrm{v}$ ) con vacilaciones gráficas que no son ajenas a otros textos castellanos, incluso en fechas posteriores a la de 1793. En el mismo documento alterna la grafia $c$ con la $z$, seguidas de vocal palatal $(e, i)$, para representar el fonema interdental fricativo sordo. Este dato, que por su perduración en los textos escritos una vez superadas las diferencias medievales entre los fonemas dentoalveolares fricativos sordo y sonoro debería ser poco significativo, sí es relevante en un texto como el que nos ocupa, por la abundancia de confusiones que encierra. $Y$ en él vamos a detenernos.

\subsection{Las grafias $c$ y $z$}

La grafia $c$ se halla en términos que en su raiz latina contenian los grupos KY, TY (también cuando van precedidos de consonante), $\mathrm{K}$ seguida de las vocales palatales $e, i$, -SK-, s- inicial y QU- latino ":

a) $\mathrm{K}+e$, $i$ : aparece en vocablos como cebada (CIBATA), cebolla (CEPULLA), celebrando (CELEBRARE), censos (CENSUS), centella (SCINTLLA), cera (CERA), cerca (CIRCA), concede (CONCIDET), concejo (CONCILIU), conducente (CONDUCENTIS), dice (DICIT), entonces (INTUNCE), Excelencia (EXCELLENTIA), Excelentisimo (EXCELLENTISSIMUS), hace (de FACERE), licencia y licenciado (de LICENTIA), lucero (derivado de LUCE), necesario (NECESSARIUS), arcipreste (del latín tardío ARCHIPRESBYTER), cierto (CERTU), cima (CYMA), cinta (CINCTA), circuito (CIRCUITUS), circunferencia (CIRCUMFERENTIA), ciudad (CIVITATE), civiles (CIVILIS), conocer (COGNOSCERE), decir (DICERE), medicina (MEDICINA), vecindario (VICINDARIUS).

b) TY y KY en posición intervocálica o agrupados con otras consonantes, en términos que en su paso al castellano han conservado la yod: abundancia (de ABUNDANTIA), audiencia (de AUDIENTIA), descripción (DESCRIPTIONIS), devoción (DEVOTIONIS), gracia (GRATIA), jurisdicción (IURISDICTIONIS), raciones (RATIONES), tercia (TERTIA).

c) -SK-: conoce (de COGNOSCERE), excepción (EXCEPTIONIS), exercer (EXERCERE), fallecen (de FALLESCERE), nace (de NASCERE), acaecido (de ACAESCERE), parece (de PARESCERE).

" Cfr. el artículo de Francisco Díaz Montesinos, "Sobre las sibilantes en el Fuero de Huete», en RFE, LXXI, 1987, págs. 287-321. 
d) S-: cierre (de SERARE) y en su compuesto encierra.

$e$ ) QUI- latino: cinco (QUINQUE), cinquenta (QUINQUAGINTA).

Por su parte, la grafía $z$ se halla en contextos similares a los de $c$ (KY, $\mathrm{TY}, \mathrm{K}$ seguida de las vocales palatales $e, i,-\mathrm{SK}-\mathrm{y}$ en términos que contenían en su étimo latino el grupo -DY-, como GAUDU > gozo):

a) $\mathrm{K}+e, i$ : arziprestazgo y arzipreste (ARCHIPRESBYTER), doze (DUODE$\mathrm{CIM}$ ), cruzan (relacionado con CRUCE), dizen (DICERE), diziembre (DECEMBRIS), prozesión (PROCESSIONIS), zelebrar y zelebrado (CELEBRARE), entonzes (INTUNCE), vezinos (VICINUM), zenteno (CENTENUM).

b) KY y TY: abraza (A+BRACCIU), adelgaza (ADELICATTARE), cabeza (CAPITIA), descalzo (relacionado con DISCALCEARE), hilanza (FILANTIA), manzano (MATTIANUS), pedazo (PITACIU), plaza (PlATTEA), poblazion (POPUlaTIONIS), San Vizente (VICENTIUS), Valenzia (VAlENTIA).

c) -GY:- goza, gozamos, gozan, gozara, gozavan (formas relacionadas con el verbo GAUDIARE).

d) También aparece la grafia $z$ en los arabismos almazén y azeite.

En otras voces la misma $z$ encierra confusiones de fonemas, como ocurre en La Codozera para nombrar al municipio de La Codosera, cercano a Alburquerque, donde el trueque de grafías va más allá del nivel de la escritura y orienta la interpretación hacia el plano de la fonología, como ejemplo claro de la confusión de sibilantes que inunda todo el manuscrito.

\subsection{Las enmiendas}

Con los mismos criterios hay que interpretar las correcciones y enmiendas que el párroco, de su puño y letra, introduce en algunas páginas para cambiar la $s$ por $z$ o al revés allí donde él mismo advierte la posibilidad de que exista una confusión. Así procede en el folio $2 \mathrm{v}$ con el término ascenso, que originalmente transcribe como azenso y, más tarde, modifica aprovechando el peculiar trazo de la $z$ para llegar a una forma ascenso en cuya caligrafía se observa el celo del escribano para no deformar en exceso la claridad de la escritura en un vocablo en el que las confusiones pasan, primero, por la simplificación del grupo culto -SK-.

La misma conducta guía la alteración que, sobre la variante inzeptos, lleva a insectos $(3 \mathrm{v})$, ejemplo útil también para observar la aspiración de las consonantes implosivas en el habla de Alburquerque hacia finales del 
siglo xviII y la ultracorrección en un contexto diferente. El folio $4 \mathrm{v}$ muestra la forma verbal subsisten como resultado de un cambio que esconde un nuevo caso de ceceo ultracorrecto: subcisten; la misma situación reflejan los términos citios (5v) debajo de sitios, aucilios bajo ausilios, sucidio bajo susidio, el verbo subsistir enmendado sobre subcistir, todos ellos en el folio $6 \mathrm{r}^{12}$, y el topónimo referente al "Sitio de la Cierba" encima de una escritura anterior con seseo, que lleva a la solución Sierva ( 7 v y 23r), donde la alteración sí resultaría relevante desde el punto de vista semántico: del femenino de 'animal mamífero rumiante cérvido, etc.' se pasaría a 'esclava, persona sometida a la autoridad de otra'. En la toponimia de Alburquerque se conserva hoy la denominación "Finca de la Cierva", que acredita el carácter erróneo de la variante sierva.

\section{Las confusiones entre los fonemas $/ \theta / \dot{y} / \mathrm{s} /$}

Frente a los ejemplos que se acaban de explicar hay otros términos en el manuscrito que escapan a la agudeza visual del escribiente y ofrecen un sistema en el que las confusiones entre $/ \mathrm{s} /$ y $/ \theta /$ a favor $\mathrm{de} / \mathrm{s} / \mathrm{son}$ un hecho habitual. La confirmación de estas vacilaciones no es nueva: ya en 1933, Tomás Navarro Tomás, Lorenzo Rodríguez Castellano y Aurelio M. Espinosa (hijo), en su artículo titulado «La frontera del andaluz» ${ }^{13}$, llamaron la atención sobre la presencia del seseo en Alburquerque y otros pueblos extremeños, y explicaron la distribución del seseo en la provincia de Badajoz con los siguientes términos:

En Badajoz se encuentra el seseo en la parte más occidental de la provincia, en una estrecha zona, de Norte a Sur, lindante con Portugal. Comprende esta zona los pueblos de Alburquerque, La Codosera y Villar del Rey, lugares situados al Norte de la capital; más al Norte, en San Vicente de Alcántara, en el límite con Cáceres, se hace distinción entre $s$ y $z$. Corresponden también a la zona de seseo Talavera la Real y la misma capital de la provincia e igualmente Olivenza, Táliga, Cheles y las aldeas de Olivenza llamadas Villarreal, Santo Domingo, San Jorge y San Benito. Fuera de esta zona se encuentra asimismo el seseo en Fuente del Maestre, muy al interior de la provincia. En los demás pueblos de Badajoz, $s$ y $z$ se distinguen con regularidad en posición inicial de sílaba y se confunden en posición final, reduciéndose a una aspiración más o menos atenuada o asimilada a la consonante siguiente (pág. 227).

\footnotetext{
12 Vid. en el "apéndice documental" de este trabajo la lámina 2, correspondiente al folio $6 r$ del manuscrito, que contiene los términos ausilios (línea 6), susidio y subsistir (línea 14).

${ }^{13} \mathrm{Vid}$. Tomás Navarro Tomás, Lorenzo Rodríguez Castellano y Aurelio M. Espinosa (hijo), «La frontera del andaluz», en $R F E, \mathrm{XX}, 1933$, págs. 225-229.
} 
Con respecto a la intensidad y la vigencia del fenómeno, los autores observaron que en las localidades de Alburquerque y La Codosera la presencia del seseo era general en todas las clases sociales, y este rasgo se aprecia, en las mismas fechas, en las respuestas que proporciona Alburquerque a las láminas del $A L P I^{14}$ correspondientes a los conceptos acero (mapa 9), azada (m. 22), brazo (m. 27), cabeza (m. 30), cazador (m. 39), caztela (m. 40), cejas (m. 4l), cepa (m. 42), cereza (m. 43), cerrojo (m. 44), cincha (m. 46), cinco (m. 47), doce (m. 71) y dulce (m. 74), donde el fonema $/ \theta /$ del castellano se transcribe con $/ \mathrm{s} /$ en palabras procedentes de étimos latinos que contenían $\mathrm{KY}$, TY y $\mathrm{K}+e, i$ y que en su evolución al castellano dieron como resultado el fonema interdental fricativo sordo $/ \theta \%$. El mismo signo emplea el Atlas para representar la $s$ en formas como ahogarse (m. 13), asa (m. 18), causa (m. 38), donde, obviamente, no se observan las confusiones inclinadas al ceceo. En la actualidad quedan restos de seseo en la franja lindante con Portugal, desde 'Alburquerque hacia el sur de la provincia de Badajoz, como prueba del influjo portugués en la fonética extremeña, y en el islote de Fuente del Maestre, donde el seseo se registra desde tiempos remotos ${ }^{15}$.

Estas noticias ayudan a justificar la presencia del seseo en el manuscrito de Alburquerque redactado para la Geografia de Tomás López. Son ejemplos similares a los que encuentra Manuel Ariza en una serie de documentos del siglo XVII procedentes de la diócesis de Badajoz, donde se leèn formas como "reconosido (ms. del Archivo Municipal de Badajoz, libro 8, fol. $53 \mathrm{v}, 9$ de agosto de 1649) y ofisio (ms. del Archivo Histórico provincial de Badajoz, legajo 315 , fol. 75,10 de marzo de 1649 )" ${ }^{16}$, idénticas a los desendientes y conosidos que escribe el clérigo extremeño en sus respuestas al cuestionario del geógrafo real.

El manuscrito, coherente con los datos del $A L P I$, proporciona una serie de casos llamativos de confusiones entre los fonemas $/ \theta / \mathrm{y} / \mathrm{s} /$ del castellano. El primer ejemplo de esta confusión se encuentra en el folio $1 \mathrm{r}$, dentro del topónimo La Codozera, repetido en varios folios del mismo documento:

\footnotetext{
14 Atlas Lingüistico de la Península Ibérica, Madrid, CSIC, 1962.

is Cfr. Antonio Salvador Plans, (cap. II: «Principales características fonético-fonológicas»), en A. Viudas, M. Ariza y A. Salvador, El habla en Extremadura, Mérida, ERE, 1987, págs. 25-37. Idénticas referencias proporciona Maria Ángeles Álvarez Martínez en el capítulo titulado «Extremeño" del libro coordinado por M. Alvar, Manual de dialectologia hispánica. El español de España, Barcelona, Ariel, 1996, pág. 177. La próxima publicación del Atlas Linguístico de Extremadura podrá arrojar nuevas luces sobre la vitalidad actual del seseo en Alburquerque.

${ }^{16}$ Manuel Ariza Viguera, «De la llamada revolución fonológica del Siglo de Oro. (Una reflexión)», en Sobre fonética histórica del español, Madrid, Arco/Libros, 1994, págs. 223-257; la cita, en la pág. 231.
} 
Es caveza de arziprestazgo que comprehende los pueblos de Villar del Rey, La Codozera y la Roca.

Por el poniente con las villas de Codozera, distante dos leguas y de su termino una y media, y del señorio de esta (folio $7 \mathrm{r}$ ).

y por lo que hace a el centeno, se extrae tambien en cambio de frutas, por los de San Vicente, Valencia de Alcantara, Salorino, Membrio, Codozera, Santiago y Herrera (20v).

El arzipreste rector y cura de las parroquias de que se ha hecho merito exerce la jurisdicion eccca. ordinaria en esta villa, la de la Codozera, Villar del Rey y la Roca (22r).

La confusión de fonemas, en estos casos, puede interpretarse como una ultracorrección favorecedora del ceceo, fenómeno que, en el marco de las hablas extremeñas, se localiza sólo en el islote dialectal de Malpartida de Plasencia ${ }^{17}$, al norte de la provincia de Cáceres. Pero es un rasgo ajeno al habla de Alburquerque y de otros municipios rayanos con Portugal, por lo cual -en un texto de este tipo-- debe tomarse como una corrección hecha por el escribano que, tal vez, seseaba y reconocía esta peculiaridad en el habla de su pueblo.

A una confusión similar obedece la forma citio por 'sitio', que aparece en el folio 8r, cuando el sacerdote escribe acerca de las veintidós leguas de jurisdicción que abarca el término municipal con sus fincas:

La tercera titulada de Malaque, principia desde la Huerta y citio de este nombre y finaliza en la de Palos.

Antes, en el folio $5 \mathrm{v}$, el pátroco emplea el mismo sustantivo, pero encima, de su puño y letra, introduce una nueva corrección para alargar el trazo de la $c$, y escribe sitio, variante general en la mayoría de las páginas del manuscrito con una doble acepción: por un lado, 'lugar ocupado por un objeto' y, por otro, 'parte de un terreno'; así se deduce de los siguientes fragmentos:

En las immediaciones de estas ruinas y varios sitios del termino se encuentran fragmentos de herrerias $(5 \mathrm{v})$.

en cuio sitio estuvieron de Guardianes S.n Juan de Prado y S.n Pedro de Alcantara (2v).

${ }^{17}$ Vid. Antonio Salvador Plans, op. cit., pág. 31. Recientemente, Carlos Canelo y Celestino Garcia confirman la pervivencia de este fenómeno en el libro titulado El habla de los chinatos, Malpartida de Plasencia, Excmo. Ayuntamiento, 1999, pág. 11. 
las aguas que por desender de sjerras y sitios arenosos son bastante delgadas (22v).

cuia mina del primero se halla al sitio titulado [...], distante un quarto de legua de la poblacion y el montano al sitio que llaman las minas (23r).

En la raya de Portugal y sitio titulado de la Cierba, a distancia de dos leguas (23r).

El fenómeno de la ultracorrección favorecedora del ceceo se observa otra vez en el sustantivo cadalzo (14v):

El Rey Don Juan el 2 la dio al Condestable y Maestre de Santiago, Don Albaro de Luna, [...] quien con su muerte, que sufrio en un cadalzo segun es notorio, perdio sus estados.

Este vocablo, según indican Corominas y Pascual, tiene su origen en el antiguo cadahalso, tomado, por conducto del catalán, del occitano antiguo cadafalcs (nominativo de cadafalc), y éste del latín vulgar *CATAFalicum, resultante de un cruce de CATASTA 'estrado en que se exponían los esclavos en venta' y fALA 'torre de madera' ${ }^{18}$. En portugués se registra la variante cadafalso con las mismas acepciones de 'tablado' o 'estrado que se levanta en un sitio público para ejecutar a los condenados a muerte', atestiguadas las dos por Machado ${ }^{19}$ y por Figueiredo ${ }^{20}$, entre otros, en sus respectivos diccionarios. En ninguna de las lenguas romances peninsulares se recogen formas con $z$ similares a las del manuscrito extremeño de 1793.

El término Ravaza (folio 8v) se menciona como nombre del municipio donde nace el río Gévora, que en los mapas modernos se identifica como Rabaça y está presente en la toponimia de Guarda, Lisboa, Marvão, Odemira, Pampilhosa da Serra y Resende, según los datos de J. P. Machado ${ }^{21}$. Rabaza, con $z$, aparece en la provincia de Orense. La variante del texto extremeño, escrita también con la grafia $z$, se refiere a la aldea fronteriza cercana a Alburquerque y La Codosera ${ }^{22}$, por lo cual parece claro que se trata de una nueva ultracorrección que elimina la pronunciación dentoalveolar de Rabaça y, al menos en el nivel gráfico, la corrige para poner de relieve su carácter interdental. Lo mismo ocurre en Ascención y proseción, caso este último en el que la metátesis refuerza las confusiones:

\footnotetext{
${ }^{18}$ Vid. Corominas y Pascual, DCECH, s, v. cadalso.

19 J. P. Machado, Dicionánio etimológico do lingua portuguesa, 3 vols., Lisboa, Horizontes, 1977 (3," ed.), s.v. cadafalso.

${ }^{20}$ C. Figueiredo, Dicionário da Lingua Portuguesa, Lisboa, 1937 (14, ed.), s.v. cadafalso.

${ }^{21}$ Vid. J. P. Machado, op. cit., s. v. rabaça.

${ }^{22}$ Cfr. los articulos ya citados de Juan M. Carrasco (en especial, el número I, 1996, pág. 68).
} 
De unas cajas [...] con las que hay suma debocion, sacandolas al publico tan solamente una vez al año, que es el dia de la Ascencion, diciendose en dicha yglesia misa y sermon con Prosecion (3r) ${ }^{23}$.

Los ejemplos analizados permiten afirmar que en el manuscrito de Alburquerque el seseo convive con la ultracorrección, fruto y resultado directo de la confusión de sibilantes que reflejan transcripciones como desender por descender, "se rosa" por "se roza", "es presiso" por "es preciso" y otras que van más allá del simple trueque grafemático.

La confusión se verifica claramente en vocablos como rosar, por rozar, procedente del latín vulgar *RUPTIARE, derivado de RUMPERE 'Tomper', que en el documento de Alburquerque se emplea con la acepción de limpiar el terreno de matas y hierbas':

lo que es de monte pardo se rosa y quema, dejando los pimpollos que se encuentran a trechos de encina y alcornoque para la cria de estos $(11 \mathrm{r})^{24}$.

La documentación más antigua de este verbo en castellano data, según Corominas y Pascual, de hacia 1282, bajo la forma roçar, transcrita con la grafia $\mathcal{C}$ que representa el fonema dentoalveolar africado sordo ${ }^{25}$. El término rozar se relaciona con el portugués roçar, si biẹn en algunas regiones lusitanas se registran variantes con el diptongo ou, vinculadas a las hablas dialectales, puesto que "en portugués literario se dice roçar sin diptongo» ${ }^{26}$.

En otros casos, el seseo se da en palabras que en latín contenian el grupo SK seguido de vocal palatal $(e, i)$ : desendientes $(15 \mathrm{v})$, desender $(22 \mathrm{v})$, conosidos (17r). Voces como exepto (2r) y exepción (11r) pueden considerarse también dentro de este grupo, pese a la diversidad de las grafias. Todas ellas, salvo conocidos que simplificó el grupo sK, en su evolución al castellano han mantenido la estructura original con la adaptación fonética de la $\mathrm{K}$ seguida de $e$, que hoy tiene una articulación interdental (descen-

23 Vid. la lámina 1 del "apéndice documental" situado al final de este trabajo, que corresponde al folio 3r, líneas 9 (ascención) y 11 (proseción).

${ }^{24} \mathrm{Vid}$. en el apéndice final la lámina 3 (folio 11r), en cuya línea 4 aparece la expresión se msa.

${ }^{25}$ Recordemos cómo en el grupo pT la $p$, en posición implosiva, se asimila a la $t$ y forman una geminada que, en contacto con la yod, se palataliza y da, desde los origenes, un resultado sordo, como se deduce de las grafias que en los textos antiguos transcriben este vocablo. De hecho, Corominas y Pascual (DCECH, s. v, rozar) se inclinan por un posible étimo *RUPtIARE, "a causa de la c sorda del castellano antiguo...". Indican que Meyer-Lübke y Espinosa han corroborado esta etimología: "en efecto, la calidad sorda de la c está probada no sólo por las grafias de 1282, de López de Ayala, de APal. y de Nebr., sino también por la pronunciación actual de la prov. de Cáceres, donde el vocablo vive en la acepción 'limpiar las tierras de las hierbas inútiles antes de labrarlas"'.

${ }^{26}$ Vid. Corominas y Pascual, $D C E C H$, s.v. rozar. 
dientes, descender, excepto, excepción), salvo en aquellas hablas que confunden los fonemas $/ \mathrm{s} / \mathrm{y} / \theta /$ :

en cuios hijos y desendientes se ha continuado y se halla actualmente (15v). señoras que han dado en sus lexitimos desendientes lustre a la patria (18r). las aguas que por desender de sierras y sitios arenosos son bastantes delgadas y saludables (22v).

los conosidos en el siglo corriente de 1700 (17 r).

ni de fruta alguna, ni de Berduras, a no haver diez canteros, exepto de ajos y cebollas (2r).

con varios pedazos de buena tierra, a exepcion de algunos canchales (11r).

En presiso (22r) y proséción (3r) el seseo afecta a términos que en sus étimos latinos mostraban la consonante velar $\mathrm{K}$ seguida de vocal palatal $\mathrm{y}$ que, como es bien sabido, evolucionó igual que la yod primera: preciso, procesión. Ya se ha hablado de la metátesis de proseción, que encierra en un mismo vocablo la tendencia hacia el seseo y su ultracorrección (asocia$\mathrm{da}$ al ceceo en todo el documento). Por su parte, presiso es una forma que vuelve a garantizar que en este documento el seseo no es un fenómeno esporádico.

En el caso de Alferes (12v), que aparece en un párrafo al lado de los nombres portugueses "(Don) Dionís" y "Barcelos", ambos con $-s$ etimológica, podríamos hablar de seseo final, por la conversión de la $/ \theta /$ en $/ \mathrm{s} /$. Este cambio no se constata en otros términos, por ejemplo en el numeral diez y en su derivado diezmo que se transcriben de esta forma en todos los folios ( $1 \mathrm{r}, 2 \mathrm{r}, 8 \mathrm{v}$, etc.), frente al sustantivo arciprestasgo, derivado del arcipreste, con un sufijo -azgo > -asgo procedente del latín -ATICU, que ofrece mayor polimorfismo en las hablas vivas del sur de la Península Ibérica (-ajo, -algo, -asgo, entre otros).

Finalmente, la grafia $s$ se encuentra en el diminutivo casitas, en un fragmento que, pese a su extensión, reproducimos para entender el significado de este sustantivo en el contexto que le es propio:

En la Yglesia del Castillo deque va hecha mencion hai un Relicario de Plata, sobre dorada, de Tercia enalto, con quatro caras, cuias Reliquias que comprehende fueron colocadas en sus Respectivas casitas que tiene con su cristal por delante, en prim.o de Diziembre de 1568; de unas cajas en que se hallavan con Desmembracion y son de N, S.or Jesuchristo, su Ss.ma Madre y de Diferentes Apostoles y santos, conlas que hai una suma debocion (folios $3 \mathrm{v}$ y $3 \mathrm{r}$ ). 
Parece claro que el clérigo de Alburquerque alude aquí a unos recipientes con tapadera de cristal en los que se guardan reliquias; esta afirmación se ve apoyada por la presencia de la forma cajas, unas lineas más abajo, destinadas al mismo fin. Así pues, casitas debe interpretarse como un diminutivo de caja, voz cuyo origen se encuentra en el latín CAPSA, que dio el resultado caxa con un tratamiento en castellano del grupo -PS- $>-i s s$-, $-i x->-x$ - en el que Corominas busca las huellas del influjo catalán a partir de una variante oriental caixa ${ }^{27}$. E1 resultado casitas por cajitas encierra un trueque aislado de $j$ por $s$, pero no por ello menos significativo. Con ejemplos inversos del cambio de $s$ por $j$, Amado Alonso habla de xexeo y lo documenta, en fechas tempranas, en textos moriscos. Ilustra sus hipótesis con un testimonio de Nebrija que dice en 1492: "los moros siempre la ponen [su šin o $s h$ ] en lugar de nuestra $s$ i por lo que nosotros dezimos Señor San Simón, por $s$, ellos dizen Xeñor Xan Ximón, por $x$ " (Ortographia, cap. I) ${ }^{28}$. Los testimonios de esta confusión son frecuentes en textos de los siglos XVI-XVII y se registran con la misma intensidad que en castellano en autores portugueses como André de Resende, citado también por Amado Alonso, que "en 1553 sigue dando el xexeo de la $s$ " 29 .

El vocablo casitas de Alburquerque no explica, por sí solo, la existencia de xexeo en las hablas extremeñas durante el siglo xviI; tampoco puede interpretarse como un tipo de ultracorrección atribuible al clérigo que firma el documento, entre otras cosas porque el término casa y su plural, casas, se transcriben con $s$ en todos los folios. Habría que conocer cómo se pronunciaba en el castellano y el portugués de la época ese fonema $/ \mathrm{x} /$ procedente del grupo PS latino en CAPSA > caja, y ver si el posible debilitamiento de la fricción velar, la aspiración de la $-s$ implosiva y el seseo influyeron o no en este aparente desliz gráfico que no halla ninguna otra manifestación en el texto.

\section{CONCLuSIón}

En el manuscrito que acabamos de estudiar, los abundantes ejemplos de confusiones entre $s$ y $z$ podrian atribuirse a un error del escribano, situación habitual en tiempos pasados. Pero la reiteración del fenómeno, unida a las enmiendas que muestra el mismo cuadernillo y los testimonios de seseo que

\footnotetext{
27 Vid. Corominas y Pascual, DCECH, s. v. casa.

${ }^{28}$ Amado Alonso, "Historia del 'ceceo' y del 'seseo' españoles", en BICC, VII, 1951, págs. 111-200. Vid. también el libro de Antonio Narbona, Rafael Cano y Ramón Morillo, Las hablas andaluzas, Barcelona, Ariel, 1998, págs. 170-171.

${ }^{29}$ Amado Alonso, «Historia del 'ceceo'...", pág. 162.
} 
brindan diecinueve de las setenta y cinco láminas del $A L P I$ en este municipio fronterizo, son datos útiles para poner de relieve la importancia que esta confusión adquiere en el habla de Alburquerque y de otros pueblos extremeños lindantes con Portugal en todas las épocas.

Es cierto que los errores caligráficos de los escribanos, en el pasado, eran más frecuentes que en la actualidad. También es cierto que un cambio de grafema no sería significativo si no se encontrara repetido en un texto y en documentos que guardan con él una cierta proximidad de fechas. En este manuscrito, la proliferación de ejemplos es llamativa, así como la reincidencia del mismo fenómeno en otros documentos de la época que contienen información sobre la villa de Alburquerque y su jurisdicción.

En los umbrales del siglo XIX, los manuscritos de Tomás López se adelantan, aunque con otros objetivos e intenciones, a los métodos de exploración de las hablas vivas mediante encuestas escritas y proporcionan materiales de sumo interés para llevar a cabo estudios sobre las hablas dialectales - extremeñas, en este caso- en una etapa crucial como es el siglo xVHI, al tiempo que sostienen una vía de investigación a partir documentos que, por su escaso valor literario, han vivido ajenos al interés filológico. 
APÉNDICE DOCUMENTAL

1.

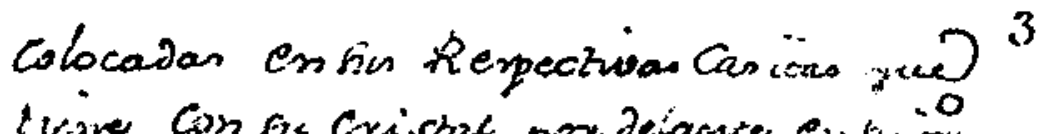
tivive cun fic Cxishac pox deiante est pim. 2e 9 izierrobe de 1568 de unas cajon errg. Setraliasan Con Desmembxaciorv; ytano

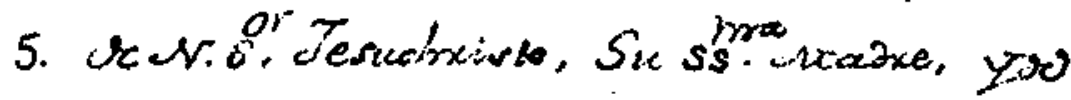
Difexenies wortoles ysantos, comlas que trai una Suma debocion, Sacarrovtas cil Publice tan stamente uma ver ic.

9. Wiv, que es ence dia dela viscencions

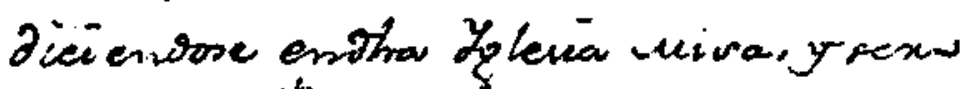

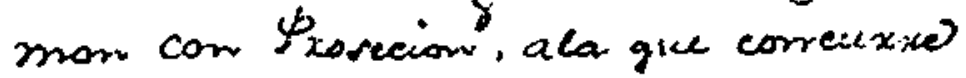
e Cuixpo Je villa y-Jemas vecing pox

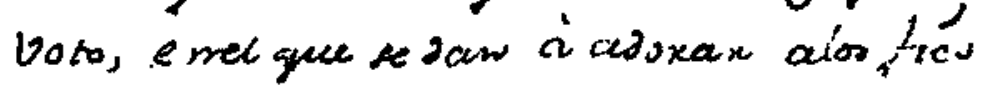
lev: $y$ dende el h.jevitayo de1760.poxo

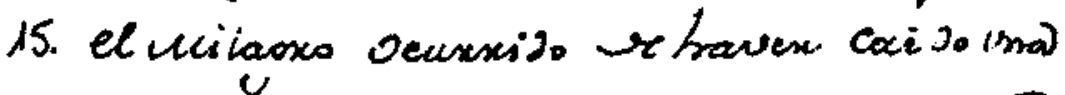
Cencellow enta toxxe del omerrafe qua

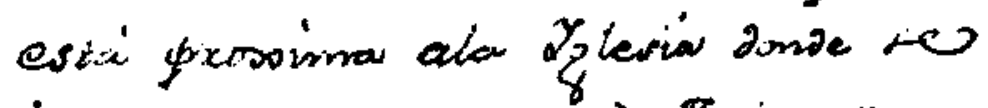
trallan Colocalas encuia goxise citava elvitmazen Jela bolboxa con mas 20. er 7oo, Rinutaies. Jeppues retraven bess maxomado wera de fien wilinemas, yins

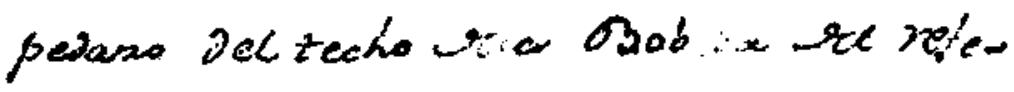


2.

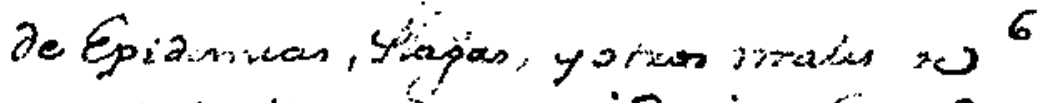

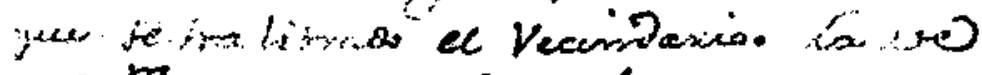

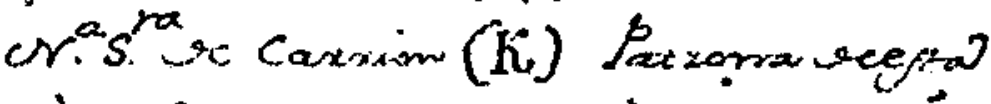

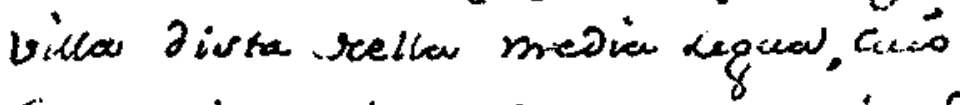

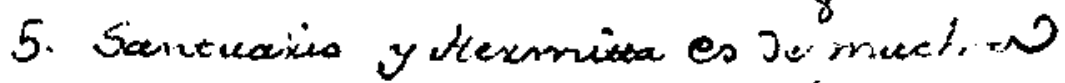

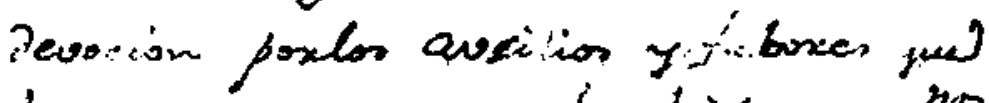

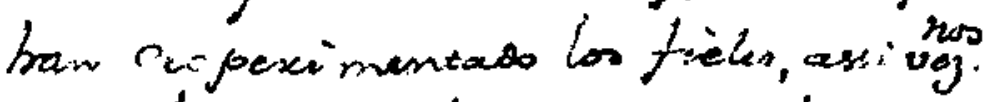
Come foxarexos, tanto renve Reyno

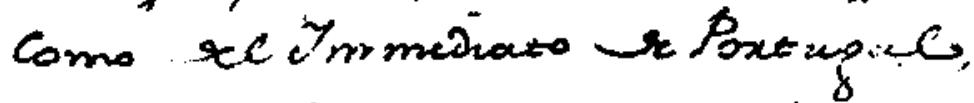
10. Ypoxto mismo es el unico objlecto vela

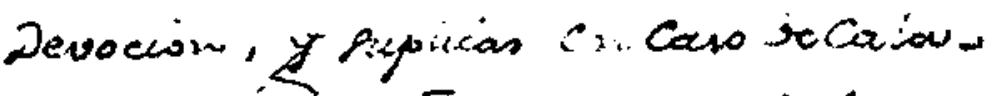

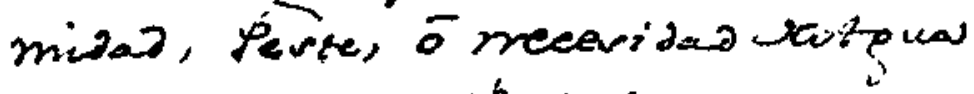

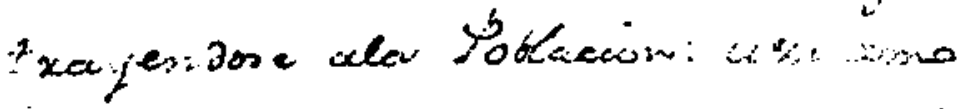

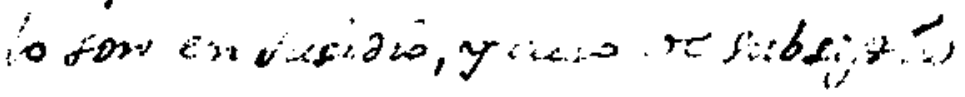

15 Sin consapuix elvtivis y focones tas

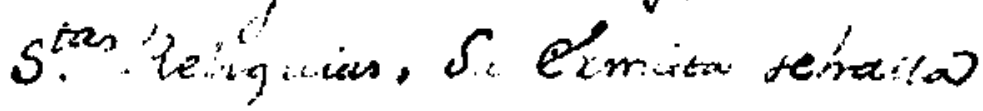

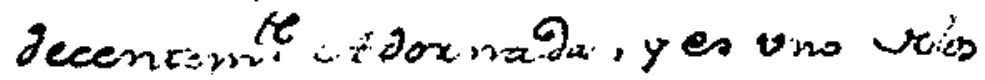

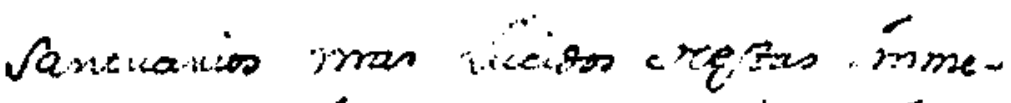

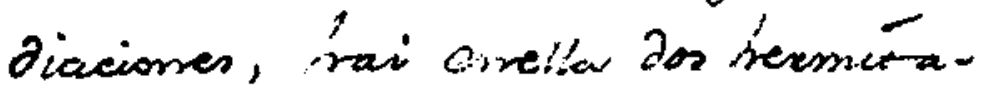
20. inos: yel Cafaluan romibirso, Teléral

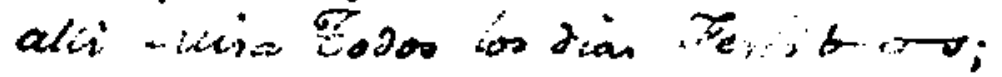


3.

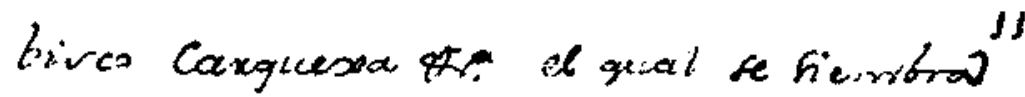

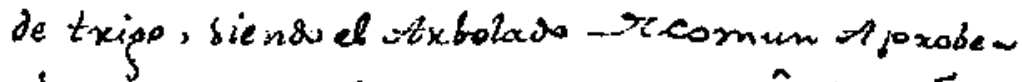

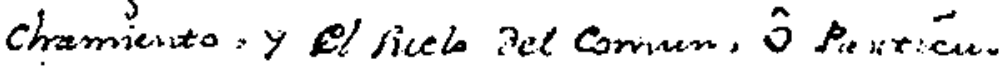

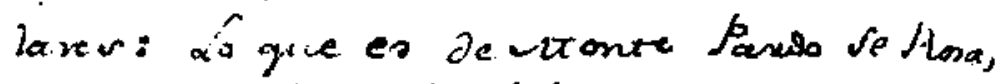

5. Vquema De,ans lor Pingottos gue fe eivers

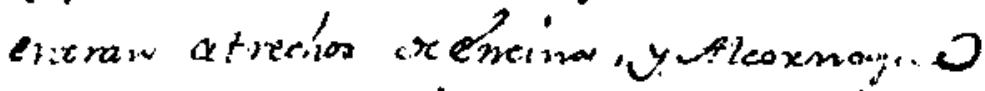
paxa a civia -reistor. La ona mitra cr6 Gexmins que cae ael voute se titula zajial. y refiembra de fenceno, is texisen sikenswo,

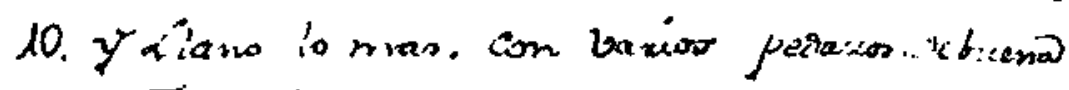

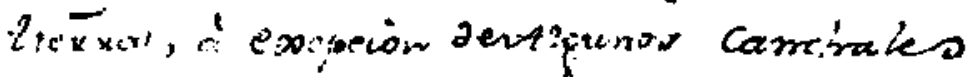

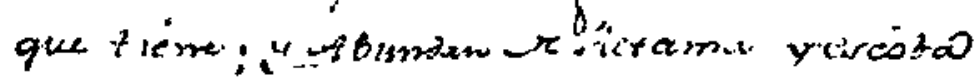
otxos paration riel.

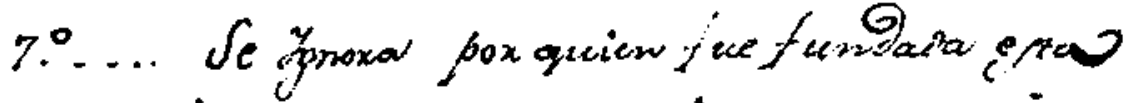

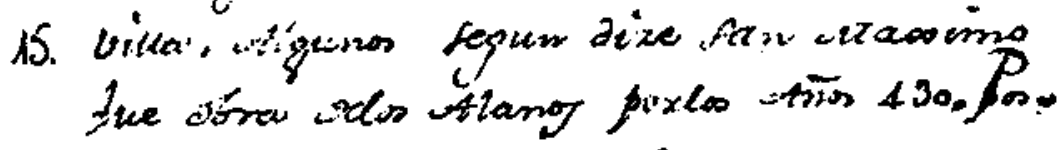

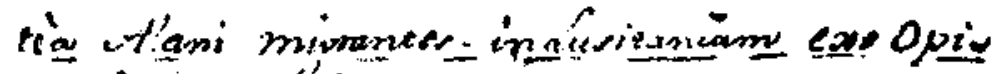
do réxobxica jecexuent itianguexum.ete its bugreexum: Aumgue orxor vieurem fox

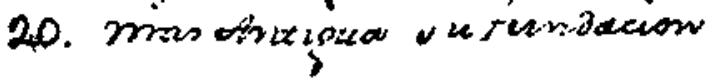

